\title{
Development of Antibacterial Fruits Wax and Its Application
}

\author{
Zhencai $\mathrm{Qu}^{1, \mathrm{a}}$ and Mengjie Zeng ${ }^{2, \mathrm{~b}}$ \\ ${ }^{1}$ Henan University of Animal Husbandry \& Economy, Henan, China \\ ${ }^{2}$ China Agricultural University, Beijing, China \\ aquzhencai@126.com, b584557839@qq.com
}

\begin{abstract}
Keywords: Cinnamon essential oil micro-capsule, Antibacterial agent, Fruit wax, Fresh-keeping Abstract. The cinnamon essential oil micro-capsules was prepared by $\beta$-Cyclodextrin $(\beta-C D)$ embedding technology, the commercially available fruit wax was used as substrate and cinnamon oil micro-capsule was added as antibacterial agent. The concentration of fruit wax, micro-capsules addition, dissolution temperature, stirring speed and other factors on the fresh-keeping effect of antibacterial fruit wax were studied through $\mathrm{L}_{9}\left(3^{4}\right)$ orthogonal experiments. The results of orthogonal experiments showed that the antibacterial fruit wax had a significant antibacterial effect on Penicillium and Staphylococcus aureus. The optimum preparation technology was determined as follows: the concentration of fruit wax was $50 \%$ (relative to the total volume of fruit wax solution), the content of cinnamon oil micro-capsules was $40 \mathrm{mg} / \mathrm{mL}$, the dissolution temperature was $70{ }^{\circ} \mathrm{C}$, the stirring speed was $700 \mathrm{r} / \mathrm{min}$, a good antibacterial effect of fruit wax solution can be obtained. The inhibition zone diameter on Penicillium and Staphylococcus aureus was $28 \mathrm{~mm}$ and $20 \mathrm{~mm}$. The fresh-keeping effect of the cherry tomato was tested, the results showed that all the indexes of the cherry tomato coated with the anti-bacterial fruit wax were better than the control group, the weight loss rate and respiration intensity were significantly reduced, and its shelf life was significantly extended.
\end{abstract}

\section{Introduction}

Fruits and vegetables are rich in nutrients required by the human body and directly affect people's health. However, due to their physiological and biochemical reactions and microbial contamination after picking, a large number of fruits and vegetables are prone to decay and deterioration during storage and transportation, resulting in huge economic losses. According to statistics, the circulating loss rate of fruit and vegetable in China is as high as $30 \%$, the annual loss of fruits and vegetables exceeds 100 billion RMB [1,2].

At present, there are many methods of storage and preservation of fruits and vegetables, including low temperature, air conditioning, radiation, anti-corrosion, heat treatment, coating storage and comprehensive preservation technology [3]. Among them, fruit wax coating preservation technology, because of its environmental safety, simple operation, and obvious effect, was widely used in the market. The cinnamon essential oil extracted from the branches and other parts of cinnamon tree had a strong antibacterial activity, when used alone, it was easy to release the volatile, reducing the antibacterial effect [4]. In this paper, cinnamon oil micro-capsule was prepared by $\beta$-cyclodextrin embedding technique, which was added into commercially available fruit wax to make antibacterial fruit wax. Through orthogonal experiment, the effects of fruit wax concentration, micro-capsule dosage, dissolving temperature, stirring speed and other factors on the preservation effect of antimicrobial wax fruit was discussed, and the fresh-keeping effect of the antibacterial fruit wax on cherry tomato was systematically studied.

\section{Experiments}

Experiment Equipment. Apparatus: Electric Thermostat Blast Drying Box (GZX-9246MBE), Water Bath Heater (HHS), Frequency Stirring Mixer (JBV-III), Workbench (HD-1360), Electronic Balance (AL204), Circulating Water-based Vacuum Pump (SHB-III), Biochemical Incubator (TF-1A), Headspace Analyzer (6000), Refrigerator (LC-162B). 
Materials: Fruit Wax (TM360), $\beta$-Cyclodextrin, Anhydrous Ethanol (AR), Penicillium, Staphylococcus aureus, Nutrition Agar (BR), Cheshire agar (BR), Cinnamon, Fresh Santa fruit, Distilled water.

Experimental Methods and Procedures. (1) Preparation of medium [5]. Bacterial culture medium: $33 \mathrm{~g}$ nutrient agar was weighed, dissolved in $1000 \mathrm{ml}$ distilled water with heating, then autoclaved at $121^{\circ} \mathrm{C}$ for 15 minutes; Fungal medium: $50 \mathrm{~g}$ chargrill was weighed, heated to dissolve in $1000 \mathrm{ml}$ distilled water, after sterilization at $116^{\circ} \mathrm{C}$ for 20 minutes, then it was bottled to use.

(2) Cinnamon essential oil extraction. $10 \mathrm{~g}$ of cinnamon power was weighed, wrapped with filter paper, then placed in a soxhlet extraction tube. $100 \mathrm{ml}$ ethanol (90\% concentration) and a small amount of zeolite was added into the flask, then heating, until the crude extract was collected to about $150 \mathrm{ml}$, the precipitate was filtered off with the vacuum filtration. The ethanol was distilled off by distillation, $5 \mathrm{ml}$ of concentrate cinnamon oil can be obtained at the conditions of $0.08 \mathrm{Mpa}, 50^{\circ} \mathrm{C}$.

(3) Preparation of cinnamon oil micro-capsules [6]. $100 \mathrm{~g} \beta$-cyclodextrin was dissolved in $500 \mathrm{ml}$ of distilled water, heated and stirred to completely dissolve. According to the ratio of core (Cinnamon essential oil) to wall ( $\beta-C D)$ of 1:7, cinnamon essential oil was dissolved in ethanol, then stirred for 2 $\mathrm{h}$ under the conditions of $80^{\circ} \mathrm{C}$ and $700 \mathrm{r} / \mathrm{min}$. After complete reaction, it was put into the refrigerator for 24 hours, then with vacuum filtration, lastly, a small amount of distilled water and ethanol was used to wash the micro-capsules. After suction filtration, it was dried at $50{ }^{\circ} \mathrm{C}$ for $4 \mathrm{~h}$.

(4) Development of antibacterial fruit wax. In accordance with the orthogonal experiment design, a certain amount of fruit wax solution and distilled water was measured. Stirring at the setting temperature, until the solution was stable, and a amount of micro-capsules was slowly added into the fruit wax solution, stirred for $30 \mathrm{~min}$.

Performance Testing. (1) Determination of antibacterial properties. Appropriate amount of antibacterial fruit wax was coated on a diameter of $7 \mathrm{~mm}$ paper, then placed on the surface of the dish coated with bacteria, placed in a $37^{\circ} \mathrm{C}$ incubator for $24 \mathrm{~h}$, and measured the inhibition zone diameter.

(2) Weight loss rate. It can be calculated according to Equation (1).

$$
\mathrm{P}=\frac{m_{0}-m_{i}}{m_{0}} \times 100 \%
$$

$\mathrm{P}$ : weight loss rate, $\mathrm{m}_{0}$ : the original quality of fruits and vegetables, mi: the quality of fruits and vegetables after storage, $\mathrm{i}$ : the storage days.

(3) Breathing intensity. It can be calculated according to the Equation (2) and (3).

$$
\begin{aligned}
& R_{C}=\frac{\left(C_{c t}-C_{c o}\right)}{100} \cdot \frac{\left(V-V_{v}\right)}{W \cdot t} \\
& R_{o}=\frac{\left(C_{o t}-C_{o o}\right)}{100} \cdot \frac{\left(V-V_{v}\right)}{W \cdot t}
\end{aligned}
$$

Rc: The amount of carbon dioxide produced, Ro: The amount of oxygen consumed, $\mathrm{mL} /(\mathrm{kg} \cdot \mathrm{h})$; Cco: the concentration of carbon dioxide before sealing, Coo: the concentration of oxygen before sealing, \%; Cct: the concentration of carbon dioxide in the closed box after sealed for $t$ hours, Cot: the concentration of oxygen in the closed box after sealed for $\mathrm{t}$ hours; $\mathrm{V}$ : the volume in the closed box, $\mathrm{mL} ; \mathrm{Vv}$ : the volume of fruit and vegetable, $\mathrm{mL}$; W: the mass of fruits and vegetables, $\mathrm{kg}$; $\mathrm{t}$ : the closed time, h.

\section{Results and Discussion}

Orthogonal Experiment. According to the experimental method, supposed the concentration of fruit wax was $\mathrm{A}$, the content of cinnamon oil was $\mathrm{B}$, the dissolving temperature was $\mathrm{C}$, the stirring speed was D. The orthogonal experiment table of $\mathrm{L}_{9}\left(3^{4}\right)$ was designed, as shown in Table 1. 
Table 1. Given factors and levels

\begin{tabular}{|c|c|c|c|c|}
\hline \multirow{2}{*}{ Levels } & \multicolumn{4}{|c|}{ Factors } \\
\cline { 2 - 5 } & (A) $[\%]$ & (B) $[\mathrm{mg} / \mathrm{ml}]$ & (C) $\left[{ }^{\circ} \mathrm{C}\right]$ & (D) $[\mathrm{r} / \mathrm{min}]$ \\
\hline 1 & 40 & 20 & 70 & 600 \\
\hline 2 & 50 & 30 & 80 & 700 \\
\hline 3 & 60 & 40 & 90 & 800 \\
\hline
\end{tabular}

Orthogonal Experimental Results. According to $\mathrm{L}_{9}\left(3^{4}\right)$ orthogonal test program, respectively tested the inhibition zone diameter under different conditions, the results were shown in Table 2.

Table. 2 Experimental schemes and results of $\mathrm{L}_{9}\left(3^{4}\right)$ orthogonal experiment

\begin{tabular}{|c|c|c|c|c|c|c|}
\hline NO. & A & B & C & D & $\begin{array}{c}\text { Inhibition zone } \\
\text { diameter of Penicillium } \\
{[\mathrm{mm}]}\end{array}$ & $\begin{array}{c}\text { Inhibition zone diameter } \\
\text { of Staphylococcus aureus } \\
{[\mathrm{mm}]}\end{array}$ \\
\hline 1 & 1 & 1 & 1 & 1 & 6 & 8 \\
\hline 2 & 1 & 2 & 2 & 2 & 11 & 12 \\
\hline 3 & 1 & 3 & 3 & 3 & 14 & 13 \\
\hline 4 & 2 & 1 & 2 & 3 & 12 & 15 \\
\hline 5 & 2 & 2 & 3 & 1 & 13 & 28 \\
\hline 6 & 2 & 3 & 1 & 2 & 20 & 16 \\
\hline 7 & 3 & 1 & 3 & 2 & 14 & 22 \\
\hline 8 & 3 & 2 & 1 & 3 & 15 & 18 \\
\hline 9 & 3 & 3 & 2 & 1 & 2 & 18 \\
\hline
\end{tabular}

Table 2 showed the best combination of antibacterial fruit wax was A2B3C1D2, the antibacterial results shown in Fig. 1.

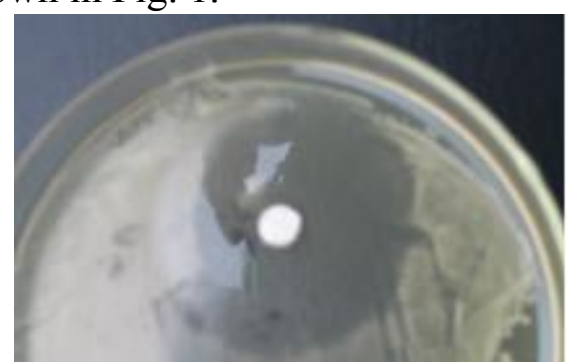

a) Antibacterial effect on Penicillium

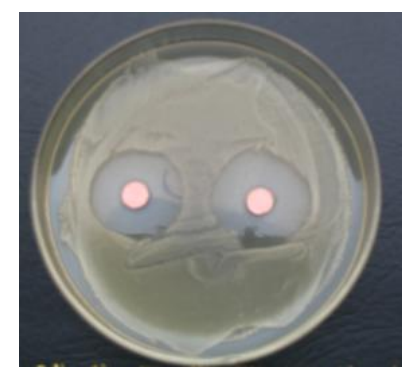

b) Antibacterial effect on Staphylococcus aureus

Fig.1 Antibacterial effect of antibacterial fruit wax on Penicillium and Staphylococcus aureus

As can be seen from Fig. 1, the antibacterial fruit wax had a significant inhibition on Penicillium and Staphylococcus aureus, the inhibition zone diameter of antibacterial fruit wax on Penicillium was $28 \mathrm{~mm}$, and $20 \mathrm{~mm}$ on Staphylococcus aureus, it showed the antibacterial fruit wax has a good antibacterial properties.

Weight Loss Rate. The use of antibacterial fruit wax can significantly inhibit the reproduction and growth of microorganisms, which will play an important role in the preservation of fruits. The weight loss rate of cherry tomato was shown in Fig. 2.

As can be seen from Fig. 2, the water content of cherry tomato was relatively high. Due to the strong transpiration, it was easy to cause a large amount of water loss during storage. The weight loss rate of the antimicrobial fruit wax group was significantly lower than that of other groups. After 18 days, the weight loss rate of the blank group reached $29.6 \%$, the weight loss rate of the fruit wax group was $14.8 \%$ and the weight loss rate of the antibacterial fruit wax group was only $9.5 \%$.

Breathing Intensity. The stronger the respiration, the more obvious decline in the quality of fruits and vegetables. Higher respiratory intensity rapidly depleted all types of nutrients in plant tissue, causing tissue destruction. The changed trend of respiratory intensity was as shown in Fig. 3. 


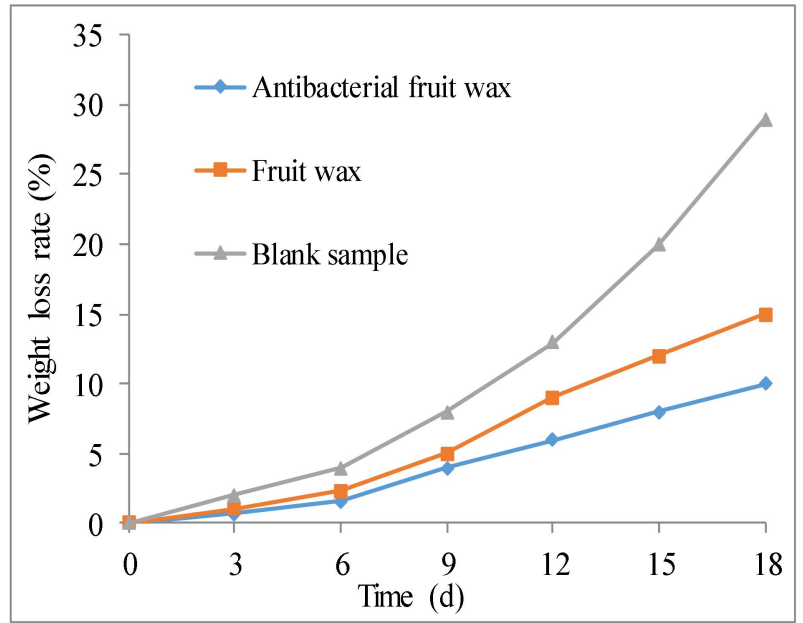

Fig. 2 The weight loss and storage time

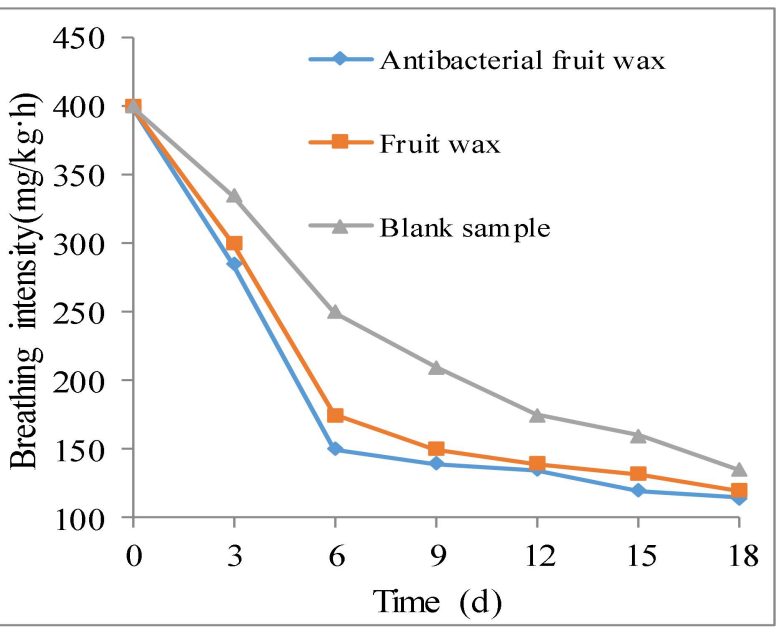

Fig. 3 The breathing intensity and storage time

As can be seen from Fig. 3, the respiration rate of cherry tomato was greatly affected by environmental factors. The respiration intensity of fresh cherry tomato was relatively high, then rapidly decreased after 6 days of storage and slowly decreased after 15 days. Compared with the control group, the anti-bacterial fruit wax group showed a significant decrease in overall respiration intensity, which indicated the antibacterial fruit wax could reduce the respiration intensity.

\section{Conclusions}

Cinnamon oil micro-capsules were prepared by $\beta$-cyclodextrin embedding technique and mixed with commercially available fruit wax to prepare antibacterial fruit wax, which had a significant inhibitory effect on Penicillium and Staphylococcus aureus. When the fruit wax concentration was $50 \%$ (relative to the total volume of the fruit wax solution), the cinnamon oil micro-capsules was 40 $\mathrm{mg} / \mathrm{mL}$, the dissolution temperature was $70{ }^{\circ} \mathrm{C}$, the stirring speed was $700 \mathrm{r} / \mathrm{min}$, a new fruit wax with good antibacterial effect can be obtained. Applying the antimicrobial fruit wax on the cherry tomato by dip coating can obviously reduce the weight loss rate and respiration intensity, prolong the storage period and increase the commodity value.

\section{Acknowledgements}

This work was financially supported by the 2017 Innovation Fund Project of Henan University of Animal Husbandry \& Economy (XKYCXJJ2017004), Zhongshan Major Public Welfare Project (2017B1022).

\section{References}

[1] J. Li, L.N. Liu, A.J. Wang: Journal of Food Science and Biotechnology Vol. 33 (2014), p. 337.

[2] Y.W. Huang, C. Xu, K. Ma: Modern Food Science and Technology Vol. 29 (2013), p. 1455.

[3] L.L. Dong, J. Deng, J.X. Tang: PACKAGING ENGINEERING Vol. 35 (2014), p. 27.

[4] W. Liu, H.J. Wang, L.W. Meng: PACKAGING ENGINEERING Vol. 38 (2017), p. 58.

[5] X.Y. Shan, L.C. Yang: Food Industry Vol. 37 (2017), p. 233.

[6] J. Deng, X.H. Tan, T.T. Liu: Journal of the Chinese Cereals and Oils Association Vol. 26 (2011), p. 89. 